\title{
Micromechanics of Twinning in a TWIP Steel
}

\author{
K. M. Rahman ${ }^{\mathrm{a}}$, N. G. Jones ${ }^{\mathrm{b}}$, D. Dye $\mathrm{D}^{\mathrm{a}}$ \\ ${ }^{a}$ Department of Materials, Royal School of Mines, Imperial College London, Prince Consort \\ Road, London $S W^{r} 72 B P, U K$ \\ ${ }^{b}$ Department of Materials Science and Metallurgy, University of Cambridge, Pembroke \\ Street, Cambridge, CB2 3QZ, UK
}

\begin{abstract}
The deformation behaviour of a TWinning Induced Plasticty (TWIP) steel was studied at quasi-static strain rates using synchrotron X-ray diffraction. A $\{111\}$ $\mathrm{RD}$ and $\{200\} \mathrm{RD}$ texture developed from the earliest stages of deformation, which could be reproduced using an elasto-plastic self consistent (EPSC) model. Evidence is found from multiple sources to suggest that twinning was occurring before macroscopic yielding. This included small deviations in the lattice strains, $\{111\}$ intensity changes and peak width broadening all occurring below the macroscopic yield point. The accumulation of permanent deformation on sub-yield mechanical cycling of the material was found, which further supports the diffraction data. TEM revealed that fine deformation twins similar to those observed in heavily deformed samples formed during sub-yield cycling. It is concluded that twinning had occurred before macroscopic plastic deformation began, unlike the behaviour traditionally expected from hexagonal metals such as $\mathrm{Mg}$.
\end{abstract}

Key words: Twinning, Synchrotron Radiation, Austenitic Steel, Yield Phenomena, Micromechanical Modeling

\section{Introduction}

High manganese content steels deform through the evolution of mechanical twins, and have therefore become known as TWinning Induced Plasticity (TWIP) steels. These austenitic steels are generally based on the Fe-Mn-Si-AlC system, where the composition is adjusted to tailor the stacking fault energy (SFE) to be within a desired range [1]. Manganese and aluminium additions raise the SFE, while the addition of silicon, in sufficient amounts acts to reduce the SFE $[5,6]$. TWIP steels exhibit extremely high strain to failure, up to $95 \%$, at tensile strengths in excess of $800 \mathrm{MPa}[1,2,3]$. This extraordinary combination of properties has led to significant interest in using these steels in

${ }^{1}$ khandaker.rahman05@imperial.ac.uk, Tel: +44 2075946811 
high strain rate energy adsorption applications, such as automotive crash safety systems and military vehicle armour [7].

The SFE is affected by temperature and composition, consequently the value is of great practical importance in face centred cubic $(f c c)$ materials, particularly austenitic steels. The SFE influences the processes of dislocation cross-slip and climb [4], which have an important role in the work hardening behaviour of a material. Low SFE results in stacking faults becoming wider, thus making dislocation cross-slip more difficult. Therefore it is essential to tailor the alloy composition for a desired SFE range.

Austenitic steels deform via a phase transformation, mechanical twinning or dislocation glide depending on the SFE [8]. At high energies $\left(\geq 45 \mathrm{~mJ} \mathrm{~m}^{-2}\right)$ deformation progresses solely by dislocation glide. Lower SFE $\left(\leq 18 \mathrm{~mJ} \mathrm{~m}^{-2}\right)$ promotes a martensitic transformation to $\epsilon$-martensite, $\gamma_{f c c} \rightarrow \epsilon_{h c p}$, which becomes a two step transformation to form $\alpha^{\prime}$-martensite at even lower energies, $\gamma_{f c c} \rightarrow \epsilon_{h c p} \rightarrow \alpha_{b c c}^{\prime}[9]$. A SFE ranging between $18-45 \mathrm{~mJ} \mathrm{~m}^{-2}$ promotes the formation of mechanical twins during deformation, which enables excellent ductility to be achieved.

Deformation twinning is a process which proceeds through a dislocation mechanism. Mahajan [10] proposed two salient features of deformation twin formation in $f c c$ crystals; firstly a three layer twin may nucleate when two coplanar $\frac{1}{2}\langle 110\rangle$ dislocations interact, and secondly a macroscopic twin may evolve when three layer twins at different levels grow into each other. Two co-planar perfect $\frac{1}{2}\langle 110\rangle$ dislocations can react either through co-planar slip or cross glide, thus the reaction; $\frac{1}{2}[\overline{1} 01]+\frac{1}{2}[\overline{1} 10] \rightarrow 3 \times \frac{1}{6}[\overline{2} 11]$, is possible. It is believed that this reaction governs the nucleation of twins in $f c c$ crystals. Furthermore in low stacking fault energy materials that twin more readily, the perfect dislocations will dissociate into Shockley partials; $\frac{1}{2}[\overline{1} 10] \rightarrow \frac{1}{6}[\overline{2} 11]+\frac{1}{6}[\overline{1} 2 \overline{1}]$. This is believed to be an intermediate step to the previous reaction as it is energetically favourable. Consequently a macroscopic twin forms when the nucleated twins grow into each other, i.e absorb more partials. This may explain why twinning is less favourable in materials with high stacking fault energies, because the partials are more tightly bound i.e. the partial dislocation pair have a narrow core width and lower mobility. The self-thickening of twins has recently been observed in-situ in coarse grained $\mathrm{Cu}-\mathrm{Ge}$ alloys and nanocrystalline $\mathrm{Ni}[11,12]$. A perfect dislocation was found to dissociate into leading and trailing partials. The leading partial subsequently splits further into a perfect dislocation and twinning partial upon encountering an obstacle. Subsequently the perfect dislocation can cross-slip and dissociate once again. This cycle continues, thereby thickening the twin on successive primary slip and cross-slip planes, without the need for the nucleation of additional Shockley partials.

Twins are observed by the formation of discrete sheared grain subregions characterised by a mirror plane at the twin interface. Consequently, the high strength and ductility of TWIP steels is due to the formation of preferentially localised twins, which lead to extensive local strain hardening. Further twinning results in an increase in the volume fraction of twins leading to a marked decrease in the mean free path for dislocation movement. This results in the 
characteristic high levels of hardening in the material. These properties mean that TWIP steels are able to meet the requirements of high formability and energy absorption.

Although research into TWIP steels is increasing, the evolution of microstructure and grain statistics, such as elastic lattice strain and texture, especially during deformation have not been investigated in detail. Therefore limited information is available within this area. Microstructure and texture evolution during deformation has been investigated by a few authors [13, 14, 15], however this has been achieved using ex-situ interrupted testing and multiple samples. Consequently, the characterisation of the twins and texture has taken place after testing and only limited bulk information could be determined. Yan et al. [16] have used in-situ synchrotron diffraction to characterise the interaction between slip and twinning during uniaxial tension. It was determined that the deformation texture is predominantly developed by dislocation gliding and that twinning impedes the reinforcement of texture.

Eshelby's $[17,18]$ self consistent approach has successfully been used to simulate the uniaxial deformation texture and lattice strain behaviour of several materials $[19,20,21,22]$. However, the application of self-consistent modelling to TWIP steels has been rather limited. Prakash et al. [23] have utilised the visco-plastic self-consistent (VPSC) model framework to evaluate twin volume fraction and compare experimental and simulated deformation texture using two different twinning models. A predominant twin reorientation [24] model and the Kalidindi $[25,26]$ approach were both evaluated, and the latter was found to predict more plausiable twin activity and provide better agreement with experimentally observed textures. Experimental texture and grain statistics have also been modelled by Yan et al. [16] using both a VPSC and elasto-plastic self-consistent (EPSC) models. The VPSC model was utilised to simulate the experimental textures, but the model textures indicated an over prediction for the contribution of twinning in the simulation. Similarly the EPSC model was used to evaluate the experimental lattice strain evolution. However, the EPSC simulation did not include a twinning scheme, consequently the contribution of slip during deformation was only modelled for the lattice strain analysis.

In the present work, the texture evolution of a TWIP steel during tensile deformation has been investigated using in-situ synchrotron X-ray diffraction. Lattice strain, peak width and intensity changes have also been examined, and the elasto-plastic self consistent model [20] has been utilised to rationalise the results. Ex-situ microscopy and cyclic tensile loading experiments were also conducted, to augment the in-situ observations.

\section{Experimental Procedures}

The TWIP steel tested (Fe-0.7C-2Al-15Mn-2Si wt \%) was obtained in $3 \mathrm{~mm}$ rolled sheet form from Tata Steel Strip Mainland Europe. The stacking fault energy of the material was determined using thermodynamic calculations to be $31 \pm 10 \mathrm{~mJ} \mathrm{~m}^{-2}$ with adjustments made to account for the silicon and aluminium contents $[27,28,5]$. 


\subsection{In-situ synchrotron diffraction}

In-situ testing was carried out on beamline ID15B at the European Synchrotron Radiation Facility (ESRF), Grenoble, France. Tensile samples, with gauge dimensions of $19 \times 1.5 \times 1.5 \mathrm{~mm}$, were tested on an Instron $5 \mathrm{kN}$ servohydraulic machine, with the tensile axis aligned to the rolling direction of the material. Samples were held in bespoke negative profile fixtures and loaded to an engineering strain of $22 \%$ in position control and at an initial strain rate of $10^{-3} \mathrm{~s}^{-1}$. Full Debye-Scherrer diffraction rings were collected using a $300 \times 400 \mu \mathrm{m}$ monochromated X-ray beam of energy $67 \mathrm{keV}(\lambda=0.1428 \AA)$ on a Pixium 2D area detector located $1046 \mathrm{~mm}$ from the sample. A sampling time of $0.4 \mathrm{~s}$ was used. The experimental setup was identical to that used in Ref [29] and testing was conducted consecutively, a schematic representation of the setup is shown in Figure 1.

The texture during deformation was reconstructed by segmenting the diffraction rings into intensity- $2 \theta$ profiles using $10^{\circ}$ interval bins around the whole ring, using the program Fit2D [30]. Instrumental parameters were obtained by using a $\mathrm{CeO}_{2}$ powder standard. The intensity- $2 \theta$ profiles were then fitted through Rietveld refinement and the texture was plotted using an Extended-WIMV (EWIMV) algorithm via the Materials Analysis Using Diffraction (MAUD) [31] program. Pole figures were visualised using the programs Pole8 and Pod2k.

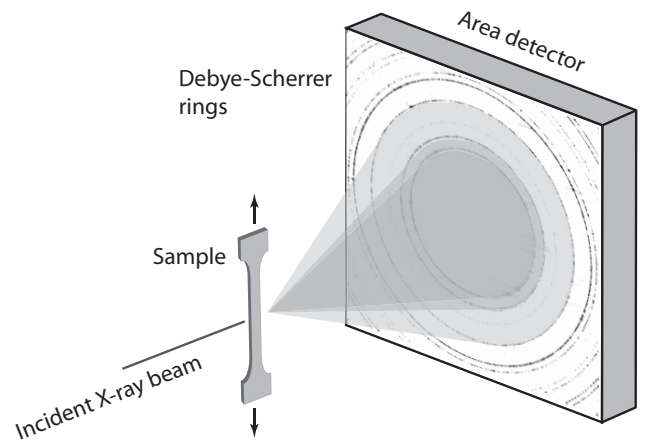

Figure 1: Schematic representation of the experimental setup for in-situ loading experiments. Figure adapted from Ref [29].

Lattice strain is represented by the lattice spacings therefore the elastic strain in a certain direction for a peak $\{h k l\}$ satisfying the diffraction condition can be determined by finding the lattice parameter $d_{i}^{h k l}$ using Bragg's law, $\lambda=2 d \sin \theta$, relative to an unstrained reference lattice parameter, $d_{0}^{h k l}$. Movement of the diffraction peaks can then be related to the elastic strain within grains in the diffraction orientation by

$$
\epsilon_{h k l}=\frac{d_{i}^{h k l}-d_{0}^{h k l}}{d_{0}^{h k l}}
$$


Therefore the shift of an $\{h k l\}$ peak along the loading direction is a measure of strain in that $\{h k l\}$ orientation.

To determine the lattice strains, individual diffraction peaks were fitted utilising a Gaussian function with the Wavemetrics program Igor Pro. $d$-spacing and intensity values for each peak were obtained from the 0 and $180^{\circ}$ tensile loading bins.

\subsection{Ex-situ tensile testing}

Ex-situ tensile tests were carried out on a Zwick Roell $100 \mathrm{kN}$ load frame and samples with gauge dimensions of $40 \times 8 \times 3 \mathrm{~mm}$ were tested, with the tensile axis aligned to the rolling direction, using an extensometer. Samples were cyclicly loaded between $10 \mathrm{MPa}$ and a selected target stress 20 times under position control at a strain rate of $10^{-3} \mathrm{~s}^{-1}$. The initial target stress was $200 \mathrm{MPa}$, this was incrementally raised by $100 \mathrm{MPa}$ after the completion of 20 cycles until the sample failed.

\subsection{Ex-situ characterisation}

Samples for optical microscopy were etched using $4 \%$ Nital to reveal the grain boundaries. A standard metallographic preparation schedule was followed.

EBSD was performed on a JEOL JSM6400 SEM equipped with an Oxford Instruments HKL Nordlys EBSD detector. Step sizes between 0.1 and $0.5 \mu \mathrm{m}$ were employed and in excess of 3000 grains were indexed for texture measurement. XRD texture measurements were made using a Philips XPert MRD machine in texture configuration via the back reflection technique using copper $\mathrm{K}_{\alpha}$ radiation. The data was then reconstructed onto equal-area projection pole figures using the preferred orientation package - Los Alamos (popLA) [32] texture analysis software package. The Philips XPert MRD machine can measure pole intensities up to an angle of $80^{\circ}$ from the sample plane normal, therefore a correction for defocussing was performed to complete the outer ring of the pole figures.

TEM analysis was conducted on a JOEL 2000FX microscope; samples were electropolished using 5 vol.\% perchloric acid and 95 vol.\% acetic acid at $30 \mathrm{~V}$ $\mathrm{DC}$ and at room temperature.

\section{EPSC Simulation}

\subsection{Self-consistent modeling}

The elasto-plastic self consistent (EPSC) model follows the single crystal plasticity model described by Hutchinson [18], which is based on the work conducted by Hill $[33,34]$. The EPSC approach treats each individual grain as an ellipsoidal elasto-plastic inclusion embedded within a homogeneous effective medium (HEM), representing the polycrystalline aggregate. The calculation procedure involved in the EPSC model is explained in detail elsewhere [20], therefore only a brief summary of the fundamental equations used in the model will now follow. Eshelby demonstrated that the stress and strain primarily 
within an ellipsoidal inclusion is constant and proportional to the applied stress and strain. Although the problem is elastic, it can be extended to the plastic regime by expressing the solution in an incremental form and introducing the concept of the instantaneous modulus, which relates the stress rate, $\dot{\sigma}$ to the total strain rate, $\dot{\epsilon}$. This forms the constitutive equation for the medium; $\dot{\sigma}=L: \dot{\epsilon}$, where $L$ is the overall instantaneous elasto-plastic stiffness. Similarly the constitutive equation for a particular grain is $\dot{\sigma}^{c}=L^{c}: \dot{\epsilon}^{c}$, where the superscript $c$ denotes an individual grain. The grain modulus $L^{c}$ is dependent on the orientation of the grain, single crystal elastic constants and plastic state of the grain.

Eshelby showed that the total strain rate in a grain can be related to that in the bulk medium via $\dot{\epsilon}^{c}=A^{c}: \dot{\epsilon}$, where $A^{c}=\left(L^{c}+L^{*}\right)^{-1}:\left(L+L^{*}\right), L^{*}$ is the effective stiffness, determined by $L^{*}=L:\left(S^{-1}-I\right)$, where $S$ is the elasto-plastic Eshelby tensor and $I$ is the fourth order identity tensor.

The total strain rate relationship provides the strain rate in an individual grain for a given medium stiffness and imposed strain rate. But, $L$ is dependent on the response of individual grains, therefore it has to be determined iteratively with the condition that the strain of the medium is equal to the weighted average over all the grains $\dot{\epsilon}=\left\langle\dot{\epsilon}^{c}\right\rangle$, thus the self-consistent equation for the bulk stiffness can be obtained; $L=\left\langle L^{c} A^{c}\right\rangle\left\langle A^{c}\right\rangle^{-1}$, while the final equation is solved iteratively. Ellipsoids of different shapes and orientations can be included.

\subsection{Simulation of TWIP steel}

The deformation of the TWIP steel was simulated to match experimental conditions, namely; a strain rate of $10^{-3} \mathrm{~s}^{-1}$ to an engineering strain of $22 \%$. The texture was modelled from a starting basis of 1000 weighted grain orientations obtained via a sample orientation distribution function using popLA, from the ESRF X-ray synchrotron starting texture measurement of the as-received material.

Twinning and slip were both modelled in the simulation. The hardening behaviour of each slip system was modelled using an extended Vocé law:

$$
\tau^{s}=\tau_{0}^{s}+\left(\tau_{1}^{s}+\theta_{1}^{s} \Gamma\right)\left(1-\exp \frac{-\theta_{0}^{s} \Gamma}{\tau_{1}^{s}}\right)
$$

where $\tau_{0}^{s}$ is the threshold stress for activation of a deformation mode, $\tau_{1}^{s}$ is the Vocé stress where the hardening extrapolates to zero, $\theta_{0}^{s}$ is the initial hardening rate and $\theta_{1}^{s}$ is the final asymptotic hardening rate for the slip system, $s$, while $\Gamma$ is the accumulated shear strain in the grain. In the present simulation, grain rotation due to slip is included, such that the texture can evolve.

Twinning was modelled using the extended EPSC code by Clausen et al. [21]. Here twinning is essentially treated as a slip system in the model, whereby a critical resolved shear stress is assigned. However, opposite twin systems are not considered since twinning is unidirectional. A new 'child' grain is created in the parent grain when twinning becomes active, the orientation of which is dependent on which twin system is being activated. The twins are allowed 
to grow and reorientate the parent grain to the new twin orientation, however this needs to be accounted for in the model, whilst keeping the overall volume fraction of the parent grain constant. The increment in twin volume fraction $\left(\delta w^{t w i n}\right)$ is given by

$$
\delta w^{t w i n}=\frac{\delta \gamma^{t w i n}}{S^{t w i n}}
$$

where $\delta \gamma^{\text {twin }}$ is the shear increment contributed by the twin system of the parent grain and $S^{\text {twin }}$ is the characteristic twinning shear. Subsequently the change in the parent grain volume can be obtained from

$$
\delta w^{\text {parent }}=-\sum_{\text {twin }} \delta w^{\text {twin }}
$$

Once a twin is created the reorientated region is treated as a new independent grain, furthermore the model allows for a whole grain to be consumed by the twin.

The stress relaxation procedure was activated within the twinning scheme to account for the contribution to diffraction by the twinned volume fractions and also provide some degree of stress relaxation in the grains due to twin activity. The procedure also requires strain compatibility at the twin interface to be satisfied.

Single crystal elastic constants for austenite $(\gamma$-Fe) were obtained from the literature [35] and are listed in Table 1. TWIP steels deform via $\{111\}\langle 110\rangle$ slip and $\{111\}\langle 112\rangle$ twinning. The critical resolved shear stress (CRSS) for each deformation mode was selected based on an approximation from the bulk stressstrain curve, these were then adjusted along with the hardening parameters until an acceptable fit for bulk stress-strain, texture and lattice strain behaviour was obtained between the model and experimental results, Table 2.

Table 1: Single crystal elastic constants in GPa for $\gamma$-Fe (austenite) [35].

\begin{tabular}{lccc}
\hline & $\mathrm{C}_{11}$ & $\mathrm{C}_{12}$ & $\mathrm{C}_{44}$ \\
\hline Austenite $(\gamma-\mathrm{Fe})$ & 276 & 173.5 & 136.3
\end{tabular}

Table 2: Fitted critical resolved shear stress and Vocé hardening parameters for each deformation system in GPa.

\begin{tabular}{llcccc}
\hline Mode & Label & CRSS $\left(\tau_{0}\right)$ & $\tau_{1}$ & $\theta_{0}$ & $\theta_{1}$ \\
\hline$\{111\}\langle 110\rangle$ & Slip & 0.26 & 0.56 & 0.36 & 0.27 \\
$\{111\}\langle 112\rangle$ & Twinning & 0.17 & 0.45 & 0.22 & 0.07
\end{tabular}



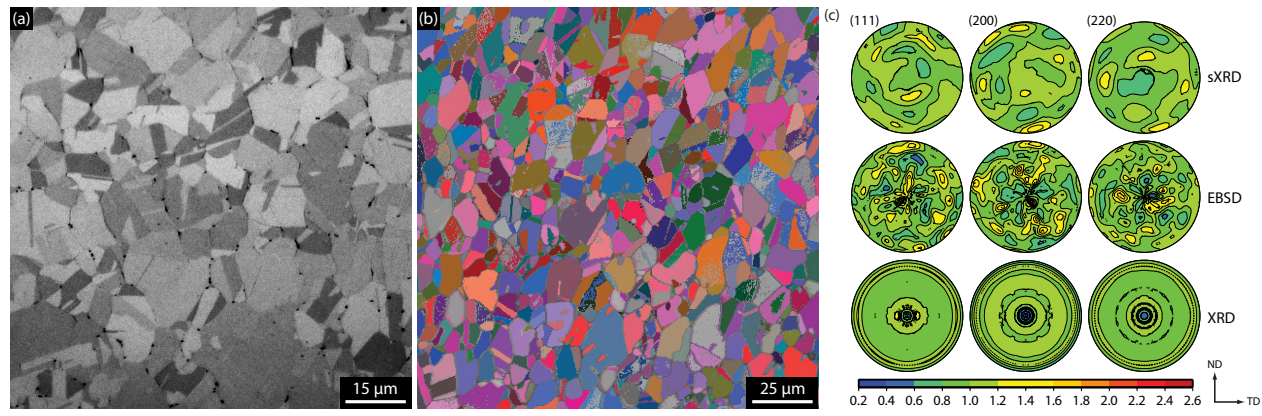

Figure 2: (a) Light micrograph of the as-received material, (b) EBSD map of the initial material [85\% indexing] and (c) weak random texture determined using EBSD, XRD and sXRD.

\section{Results and Discussion}

\subsection{Macroscopic chracterisation}

The initial microstructure of the material was single phase composed of equiaxed austenite grains, some of which contained annealing twins. The average grain size was $10 \pm 6 \mu \mathrm{m}$, which was determined using EBSD, Figure 2(a,b). The initial texture of the TWIP steel plate was weak and essentially random, Figure 2(b,c), this was confirmed using three experimental techniques, namely electron backscatter diffraction (EBSD), lab X-ray diffraction (XRD) and synchrotron X-ray diffraction (sXRD). The conclusion of an initial random texture from three separate experimental techniques provides a sound basis for the reconstruction of the synchrotron X-ray diffraction data, since it implies that Rietveld refinement of the data does not induce any false texture.

The bulk stress-strain curve for the material tested to $22 \%$ engineering strain is shown in Figure 3. A yield of approximately $460 \mathrm{MPa}$ was observed and the yield transition occured gradually. The elastic modulus of the material was $170 \mathrm{GPa}$. An almost linear true hardening rate of $\sim 2.3 \mathrm{GPa}$ is exhibited, indicating a high level of strain hardening. A high linear hardening rate is often observed in austenitic steels that deform through twinning, as this is usually associated with the interaction between dislocation gliding and twinning [36]. The formed twins act as strong barriers to dislocation motion and further straining results in a greater volume fraction of twins being created. This effectively results in a continuous grain refinement process i.e. a dynamic Hall-Petch effect where the mean free path for dislocation gliding is continously reduced [37, 13].

The tensile behaviour predicted by the model shows excellent agreement with the experimental results and the gradual yielding and linear hardening rate is also well replicated. The predicted activity of the deformation modes suggested a sharp increase in twinning activity at the onset of plastic deformation, which gradually decreases with further deformation. The sharp increase in twin activity may be interpreted as the nucleation of twins in the material when plastic deformation initiates. The slow decrease in activity with further 


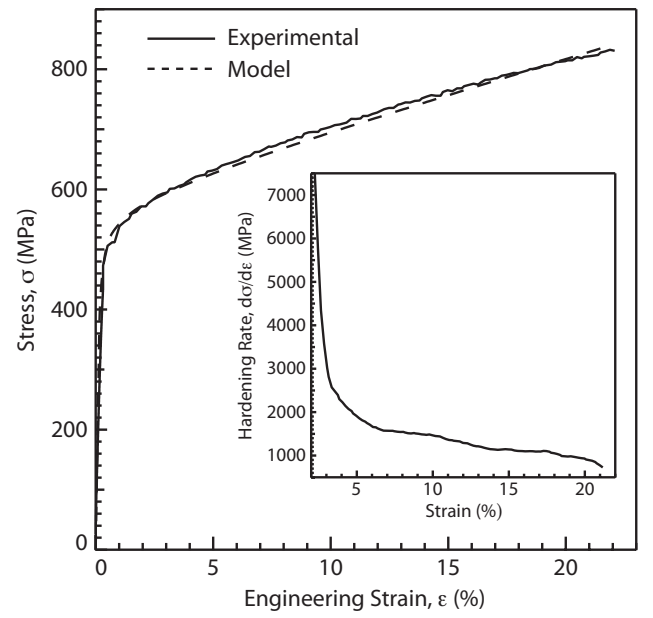

Figure 3: Macroscopic stress-strain and hardening (inset) curves showing the experimental (solid line) tensile behaviour and the model prediction (dashed line).

deformation may indicate a cessation in twin nucleation and the initiation of twin thickening and subsequent twin growth within the model framework.

\subsection{Lattice strain and peak width evolution}

The evolution of lattice strain during loading is shown in Figure 4, along with the predicted model results. Figure 4 also shows the lattice strain behaviour of an austenitic 304 stainless steel, which was tested using an identical experimental setup during consecutive tests (data from Ref [29]). The $\{111\}$ orientation is the stiffest orientation, which is followed by the $\{220\},\{311\}$ and $\{200\}$. Furthermore the $\{200\}$ and $\{311\}$ orientations are observed to remain elastic the longest. This behaviour can be seen more clearly in the simulated results, Figure 4 (solid black lines). The diffraction elastic constants remain consistent with that observed in other austenitic steels [38], whereby the order of the stiffest orientations are as expected. The model successfully predicts the general behaviour of the orientations, however the simulated results do deviate from the experimental data. The disparity between the experimental and simulated lattice strain can firstly be explained due to the changes observed in the experimental lattice strain below the yield point which are difficult to replicate within the model. Secondly, the model prediction is a compromise fit between macroscopic stress-strain, texture and lattice strain and finally the model predicts twin activation to occur later than the experimental evidence would indicate. Consequently, simply activating twinning earlier in the model would result in orientations yielding prematurely and texture strengthening, however this would not result in the observed sub yield deviations in the lattice strains.

The $\{200\}$ orientation begins to gradually yield below the macroscopic yield point, which is indicated by the inflections observed at $\sim 100$ and $200 \mathrm{MPa}$. This 


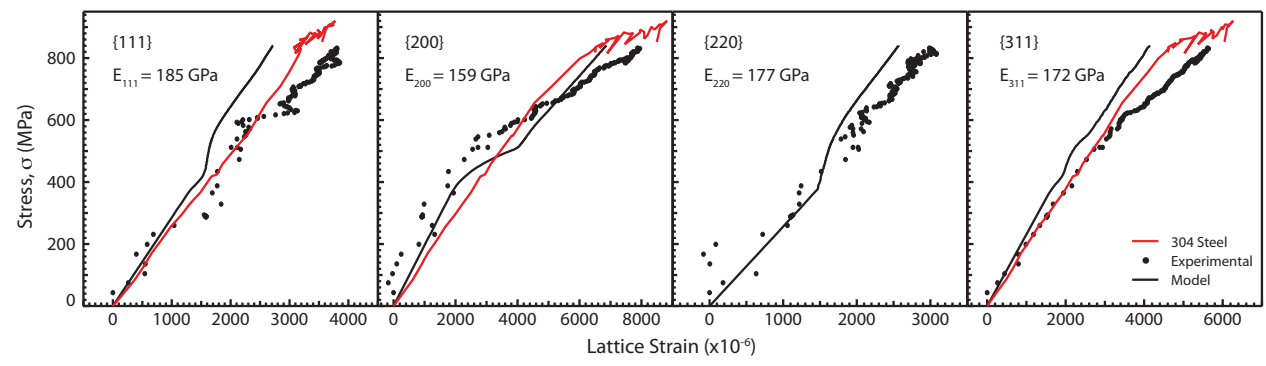

Figure 4: Experimental and simulated lattice strain evolution during tensile loading of the TWIP steel compared to the lattice strain evolution of an austenitic 304 stainless steel. 304 data from Ref [29], sample texture from prior forming operations prevented diffraction from the $\{220\}$ orientation.

would indicate that micro-plasticity occurs in this orientation before macroscopic yielding. Although micro-plasticity can occur before macroscopic yielding, the experimental results indicate this occurs at a stress significantly lower than the yield point. The $\{111\}$ and $\{220\}$ orientations exhibit a similar behaviour, which suggests the initiation of non-linear behaviour before the macroscopic yield point for all these orientations and micro-plasticity. The onset of plasticity in three orientations at stresses below the yield point cannot be replicated using the self-consistent model and they are not observed in the 304 stainless steel. However the model does indicate plasticity occurs at a slightly lower stress than the yield point. This implies that the material is not truly elastic before the yield point. Small inflections are seen in the lattice strain profiles once the macroscopic yield point is exceeded, this can be replicated using the model and be clearly seen in Figure 4. After macroscopic yielding the grains become plastic and consequently they are no longer able to accrue elastic load at the same rate as when the material was macroscopically elastic. This usually corresponds to an inflection in the lattice strain profile around the yield stress of the material. The inflections are the result of load partitioning from orientations that have plastically deformed to those that have not. The experimental evidence shows that the $\{111\}$ and $\{220\}$ orientations are the first to exhibit such a reduction in the rate of accumulating lattice strain as deformation progresses, while the $\{311\}$ and $\{200\}$ orientations are most capable of accumulating further strain. Therefore as further deformation progresses, grains in the $\{311\}$ and $\{200\}$ orientations accumulate the majority of the corresponding lattice strain. A non-linear increase of lattice strain is clearly apparent for the $\{200\}$ orientation, this orientation is also able to accrue greater lattice strain compared to other orientations, thus further indicating load partitioning between the orientations.

An obvious feature in the experimental lattice strain, Figure 4, are the deviations from linearity, particularly below the macroscopic yield point. This can be clearly seen for the $\{111\},\{200\}$ and $\{220\}$ orientations, where a $-170 \mu \epsilon$ deviation is observed for the $\{111\}$ and a $-740 \mu \epsilon$ is observed for the $\{220\}$ ori- 
entations at $\sim 120 \mathrm{MPa}$. This value is very similar to the twin initiation stress predicted for the experimental material by the authors in a separate study [39]. Similar behaviour has been seen in Ref [16], particularly for the $\{220\}$ orientation. However, the authors attributed this behaviour to be due to error bars caused by poor diffraction sampling of the grains in the longitudinal direction. In the present study, it is believed that these deviations are due to intrinsic changes within the material and not because of poor diffraction sampling. The experimental setup was identical to that in Ref [29], which was on a single phase austenitic stainless steel; the two materials were also tested consecutively. Deviations were not observed in the experimental lattice strains of the stainless steel and a change in diffraction sampling between the tests is highly unlikely.

The austenitic 304 steel which was of similar grain size tested in Ref [29] deformed solely by slip and the lattice-strain behaviour did not present any similar deviations such as those observed for the TWIP steel. Therefore, the possibility of deformation via slip causing the deviations in unlikely as this is not observed in the case of the 304 stainless steel. Thus it is suggested that the deviations in the lattice strain profile is due to twinning occurring in the material. An incubation plastic strain in polycrystals is often required before twinning is observed, which is conventionally attributed to either a requirement to raise the operative critical resolved shear stress for slip e.g. by forest hardening, above that required for twinning or to form a nucleating population of partial dislocations. The twinning stress is usually found to be temperatureindependent. In addition, the stress in the surrounding matrix will be reduced by the twin. However, the lattice strain behaviour indicates twinning operating at low stresses and more importantly below the macroscopic yield stress. The strain relaxation associated with the deviations further suggest the initiation of twinning in the material, as once a twin has nucleated strain relaxation in the surrounding matrix is to be expected. Similar lattice strain behaviour has been observed in zirconium alloys [40,22] and magnesium [41], where deviations in the lattice strain are present during deformation, particularly for grains in the $\{0002\}$ orientation. Among the suggested causes of these deviations, twinning has been suggested as the most plausible contributing factor.

Diffraction peak widths are affected by crystal size and the presence of lattice defects such as the accumulation or nucleation of dislocations and twins. During deformation peak broadening may occur due to many reasons. An inhomogeneous strain field in the scattering volume, such as an increasing density of dislocations in the grains can produce peak broadening, also reducing the scattering size through twinning or having varying strains from different grains will produce peak broadening. Similarly, increasing the crystallite size, e.g. through twin growth, will produce an opposite effect and result in peak narrowing. The $\{111\}$ peak width evolution, Figure 5, indicates large variations in the peak width at low stresses, which coincide with the deviations seen in the lattice strain behaviour. It is suggested that the increase in peak width is due to twinning, which causes a reduction in the crystallite size. Similarly, as the twins grow, peak narrowing occurs as observed in Figure 5. Using the Scherrer equation and an approximation of the shape factor for a lenticular morphology 
crystallite $(\mathrm{K}=1.34)$, the ideal crystallite size i.e. twin thickness, required to produce the observed variations in the full width half maximum of the $\{111\}$ peak, was calculated to be $\sim 5-10 \mathrm{~nm}$. Since the nucleation of large densities of dislocations are not expected at these low stresses it is assumed that the fluctuating peak widths are being influenced by twinning. The peak width begins to increase at a constant rate once yielding is fully established, i.e. beyond $600 \mathrm{MPa}$. This steady increase may be due to an increase in the density and accumulation of dislocations post yielding. Smaller deviations i.e. peak narrowing, are subsequently observed, which also coincide with the deviations observed in the lattice strains. These may be resultant of further twin growth. Since the rate of peak broadening does not change or exhibit a plateau as deformation progresses, it can be assumed that the cessation of twinning has not occurred at $22 \%$ strain. It is suggested that the fluctuating peak width behaviour seen at lower stresses may be due to the nucleation of twins, since the twins act to reduce the effective grain size in the material, consequently this contributes to a peak broadening effect. Therefore, the constant peak broadening after $\sim 600 \mathrm{MPa}$ may suggest the cessation of twin nucleation and the initiation of twin thickening via the absorption of partial dislocations. The 304 stainless steel tested in Ref [29] does not exhibit a similar behaviour to the TWIP steel; during elastic loading the $\{111\}$ peak width essentially remains constant, Figure 5.

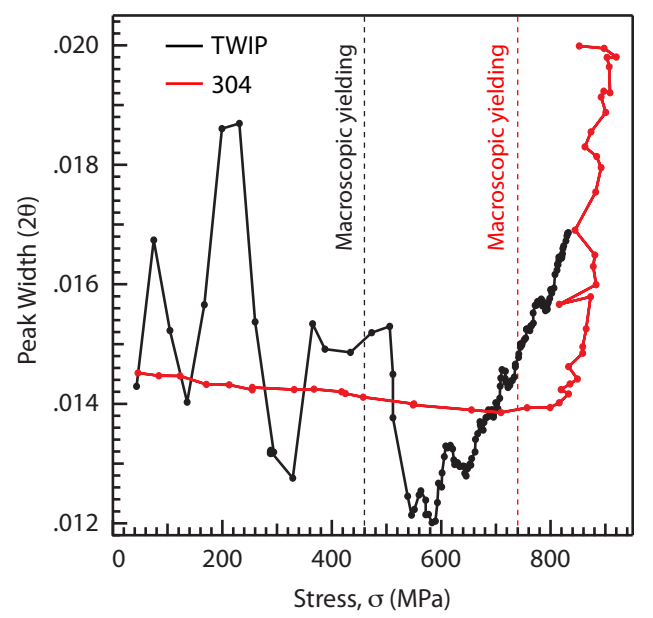

Figure 5: $\{111\}$ peak width evolution in the tensile direction during loading of the TWIP and 304 steels.

\subsection{Texture evolution}

The final experimental deformation texture and the model prediction are shown in Figure 6. The weak starting texture intensified in the loading direction particularly on the $\{111\}$ pole figure, but also on the $\{200\}$. The texture has also developed a distinct four-fold symmetry. Furthermore, the final tex- 
ture predicted by the model shows good agreement with the experimental pole figures.

There is a pronounced fiber texture, which has developed in the $\langle 111\rangle / / \mathrm{RD}$ and a weaker $\langle 200\rangle / / \mathrm{RD}$ component. The final texture has four main components, the most intense being $\{110\}\langle 112\rangle$ brass, followed by $\{110\}\langle 110\rangle$ rotated Goss, then $\{112\}\langle 110\rangle$ rotated copper and finally a $\{001\}\langle 100\rangle$ cube component.

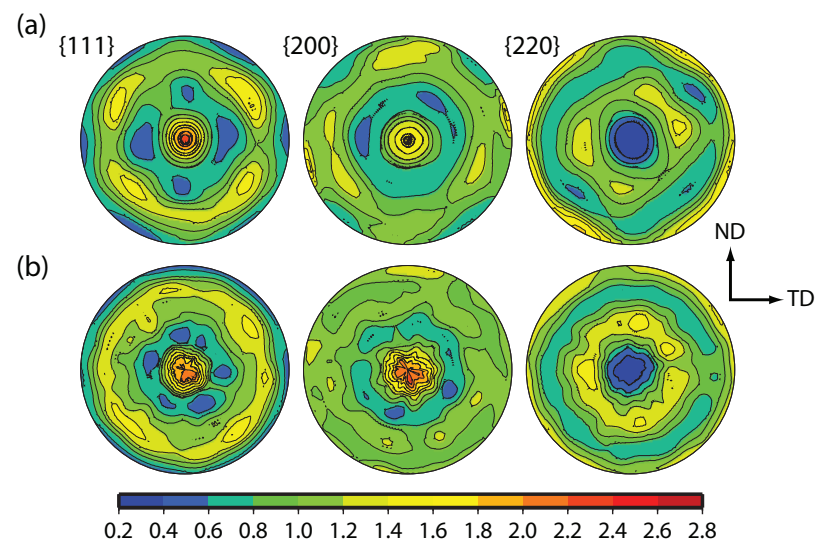

Figure 6: Final texture after deforming to $22 \%$ in the rolling direction, (a) experimental and (b) simulated (tensile direction out of page).

The evolution of the $\{111\}$ pole figure texture during deformation and the corresponding predicted texture by the model is shown in Figure 7. The texture begins to sharpen and a distinct four-fold symmetry begins to form by $\sim 7 \%$ strain. Furthermore, stronger variations in intensity begin to initiate from this point. Small reorientations of the pole figures are observed at low strain i.e. $<0.3 \%$ strain, however these are not predicted in the simulated results. It should be noted that these reorientations predominantly occur below the macroscopic yield point of the material, therefore the model is unable to predict any slip or twin activity. The experimental observation would suggest possible twin activity or twin nucleation, which result in small reorientations in the pole figures.

The strengthening of the $\langle 111\rangle / / \mathrm{RD}$ and the main texture components during deformation indicates that twinning activity is increasing. This would concur with evidence that twinning is well suited for the $\langle 111\rangle / / \mathrm{RD}$ fiber component because the Schmid factor for twinning is greater than that for slip [14, 42]. Similarly the weaker $\langle 200\rangle / /$ RD fiber is better suited for slip, which is apparent in the experimental textures. The slight over prediction by the model for the intensity in the tensile axis of the $\{200\}$ pole figure, would indicate an overestimation for slip activity.

Twinning generates new orientations, which can also modify texture, therefore the texture evolution would indicate twin nucleation even at the higher strains. However, after $11.85 \%$ strain new texture components are not observed and only strengthening in intensity occurs, i.e. the $\{111\}$ gets bigger with fur- 


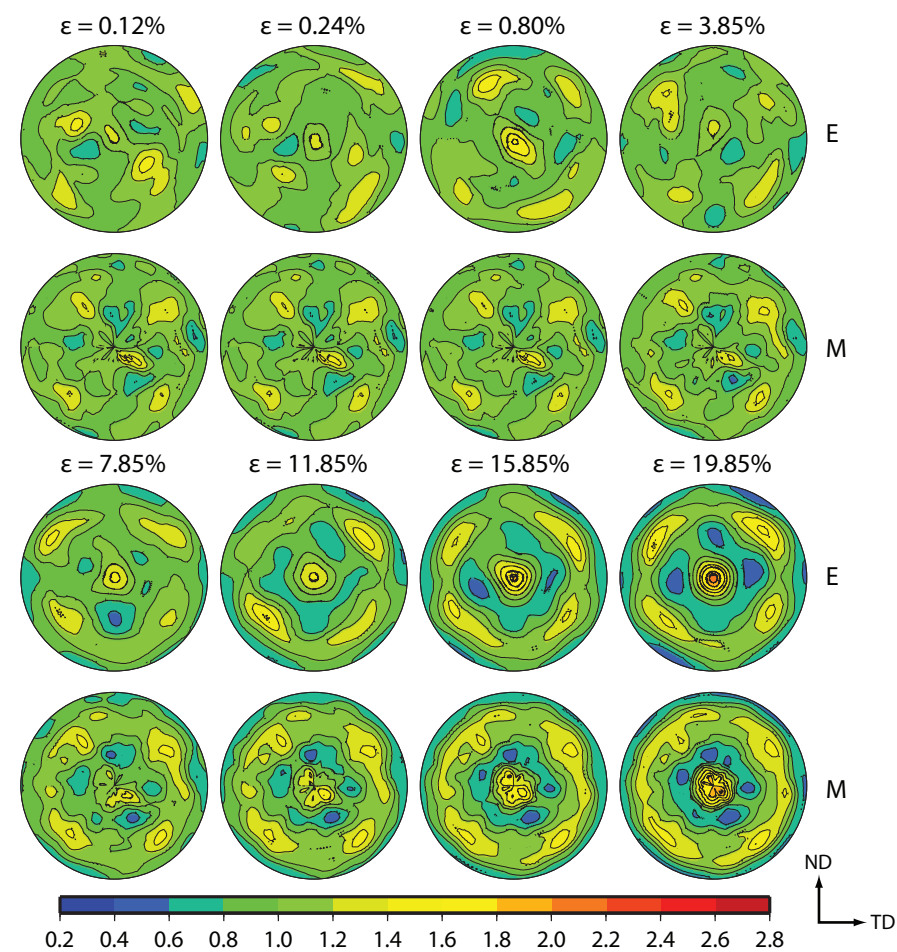

Figure 7: $\{111\}$ pole figure projection of the experimental $(\mathrm{E})$ and modelled $(\mathrm{M})$ texture evolution during tensile loading to a final strain of $22 \%$.

ther strain. However, some authors have suggested that the overall contribution of twinning to the deformation texture is minimal because the twins formed are very fine.

The texture index, Figure 8, indicates strengthening of the texture throughout the deformation process. A small increase in strength is detected during elastic straining, which is followed by a steady strengthening of the texture to $\sim 16 \%$ strain. The rate at which strengthening occurs increases after $16 \%$ strain; the experimental pole figures show that after this strain no new texture components are formed, and further deformation only strengthens the existing texture components. Consequently this strengthening results in an increase in the texture index. The strength of the simulated textures are generally higher than the experimental observations. This is often observed in self consistent and Taylor type texture models.

The $\{111\}$ RD peak intensity evolution, Figure 9, generally exhibits an increase in the intensity during deformation. This is in contrast to the 304 austenitic steel tested in Ref [29] where intensity decreases with progressive deformation. A distinct feature of the intensity evolution is the sharp changes in intensity seen during deformation. The intensity fluctuations are even observed at nominally elastic stresses and furthermore coincide with in the lattice strain 


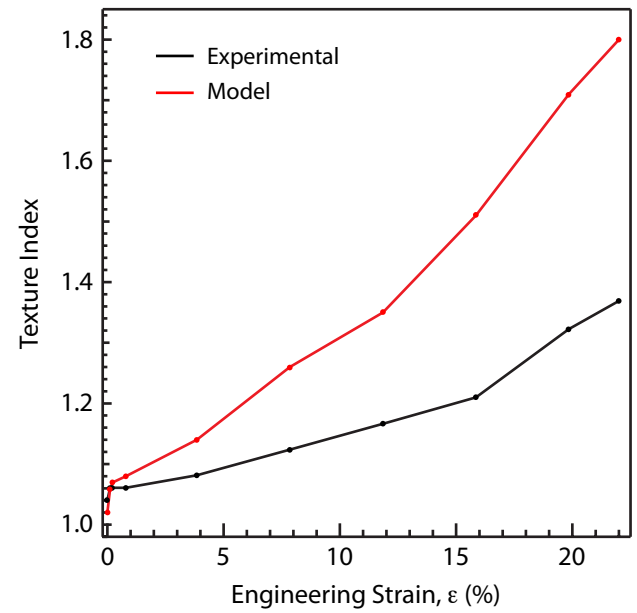

Figure 8: Experimental and model evolution of texture index with progressive deformation.

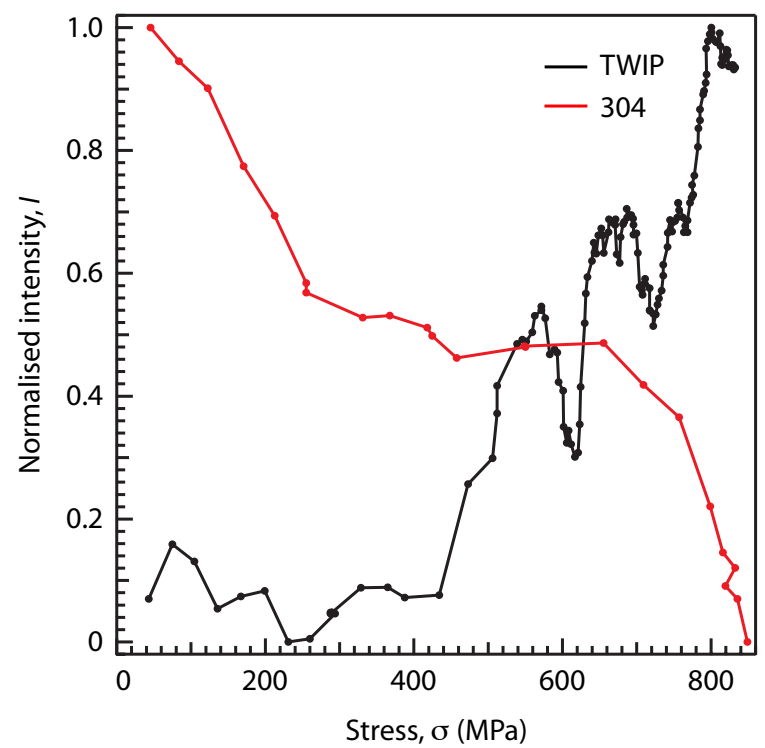

Figure 9: Evolution of the normailised $\{111\}$ RD intensity in the tensile direction with applied stress compared to 304 stainless steel [29].

deviations seen in Figure 4. Abrupt changes in diffraction intensity suggest a change in the orientation of the diffracting volume, which can be caused by deformation twinning. Therefore the sharp changes in diffraction intensity can be attributed to twinning, while the gradual increase in intensity is due to gradual grain rotation during deformation which can be attributed to slip mechanisms. 


\subsection{Sub-yield twinning: cyclic testing and microscopy observations}

The post-deformation microstructure is shown in Figure 10; profuse twinning is observed within the grains. These can then be observed using EBSD, which shows the presence of annealing twins and also, using the band contrast, the presence of very fine twins within many of the grains, particularly those in the $\{111\}$ RD orientation, as indicated with an arrow in Figure 10(b). EBSD also highlights that whilst many of the grains are in the $\{111\}$ RD orientation, many of the grains are not; similarly the fine twins are observed only in some of these grains. Figure 11 shows low magnification (a) and high magnification (bright field) views of the twins in the post-deformed sample. Consistent with Idrissi et al. [43], the twins are on the order of $20 \mathrm{~nm}$ in thickness and are filled with faults or smaller microtwins. In addition, the matrix around the twins is relatively free of dislocations. The selected area electron diffraction (SAED) patterns indicates that two different twin systems exist, type one forming on the (111) plane and type two forming on the $(\overline{1} 1 \overline{1})$ plane.

In order to verify the implication from the diffraction measurements that twinning occurred below the macroscopic yield stress, some additional mechanical testing was performed. Figure 12 shows the effect of cyclic loading for 20 cycles at stresses of 200,300 and $400 \mathrm{MPa}$, all below the macroscopic yield stress. It is observed that the sample progressively deformed, with a substantial residual strain being imparted to the sample; at $400 \mathrm{MPa}$ this was $\sim 8 \mu \epsilon$ per cycle. The accumulation of plastic strain during cyclic loading was also corroborated using a sample with a strain gauge mounted to rule out artefacts from the extensometer. The strain gauge also indicated an accumulation of permanent strain with each cycle.

Figure 13 shows the first unload and reload of each cycle, indicating that substantial hysteresis occurs in each case, in addition to the residual plasticity imparted. Also it can be seen that there was some relaxation that occurred during the very final stages of unloading.

Therefore even macroscopically it can be concluded that plastic deformation occurs at stresses as low as $200 \mathrm{MPa}$, less than half of the nominal $0.5 \%$ offset yield stress of $480 \mathrm{MPa}$.

Attributing these to twinning is difficult because the twin density is rather low, but thin deformation twins around $5 \mathrm{~nm}$ in thickness could be observed in TEM (Figure 14) after performing 1000 cycles on a sample between 10 and $200 \mathrm{MPa}$. This shows the end of a very fine twin that decomposes into the stacking faults that form the twin walls. The twin thickness observed using TEM is within the predicted range calculated using the Scherrer equation that would produce the observed sub-yield peak width broadening observed.

Therefore it can be concluded, from TEM, macroscopic testing, from the lattice strain, diffracted intensity and peak width profiles, that twinning occurs below the nominal yield stress in this material. 


\section{Conclusions}

The texture evolution and micromechanical response of an initially untextured TWIP steel have been studied during quasi-static tensile straining along the rolling direction at $10^{-3} \mathrm{~s}^{-1}$ using in-situ synchrotron diffraction and post hoc microscopy. The following conclusions can be drawn

1. Experimental evidence from synchrotron X-ray diffraction lattice strain evolution, peak width evolution, cyclic testing using an extensometer and strain gauge coupled with TEM observations indicate that sub-yield twinning occurs.

2. Elasto-plastic self consistent modelling has been used to capture the texture and lattice strain evolution during loading.

3. The $\{111\} \mathrm{RD}$ and $\{200\} \mathrm{RD}$ texture evolved continuously from the very earliest stages of deformation.

4. The flow curve and texture evolution could be satisfactorily modelled using a self-consistent micromechanical model.

5. The lattice strain response show unusual deviations below macroscopic yield, a feature not observed in a similar grain size austenitic stainless steel using an identical experimental setup.

6. Corresponding deviations were also observed in the peak width and intensity; these are not felt to be artefacts as conventional linear behaviour is recovered in the later stages of deformation.

7. The very fine twins expected of a TWIP steel were observed after deformation, such that twinning can occur continuously and provide work hardening up to large strains.

8. Macroscopic cyclic testing indicated that sub-yield permanent deformation occurred even at $200 \mathrm{MPa}$, a stress less than half the macroscopic yield stress.

9. TEM examination indicated that deformation twins were induced by this cyclic testing.

10. Therefore, it is concluded that twinning can occur in the very earliest stages of deformation in the material studied, prior to the buildup of a large population of partial dislocations. However, the microscopy indicated that these twins were still associated with partial dislocations.

\section{Acknowledgements}

The authors would like to thank Thomas Buslaps and Jerome Andrieux on ID15B for their assistance and the ESRF for funding experiment MA862. J.L.W. Warwick and V.A. Vorontsov at Imperial College London are acknowledged for their assistance. This work was supported from the materials and structures research programme delivered by Team MAST for the Defence Technology and Innovation Centre, part of the UK Ministry of Defence. The support of Tata Steel is gratefully acknowledged, both in useful discussions, particularly with PF Morris, M Cornelissen, PA Davies and B Berkhout. 


\section{References}

[1] Frommeyer G, Grassel O. Mater Sci Tech 1998;14

[2] Frommeyer G, Brux U, Neumann P. ISIJ Int 2003;43:438

[3] Grassel O, Kruger L, Frommeyer G, Meyer L. Int J Plast 2000;16:1391

[4] Schramm R, Reed R. Metall Mater Trans A 1975;6:1345

[5] Oh B, Cho S, Kim Y, Kim Y, Kim W, Hong S. Mater Sci Eng A 1995; 197:147

[6] Dumay A, Chateau J, Allain S, Migot S, Bouaziz O. Mater Sci Eng A 2008; 483-484:184

[7] Rahman K M, Vorontsov, Dye D. Mater Sci Eng A 2014;252-261:589

[8] Allain S, Chateau J, Bouaziz O, Migot S, Guelton N. Mater Sci Eng A 2004;387:158

[9] Curtze S, Kuokkala VT. Acta Mater 2010;58:5129

[10] Mahajan S, Chin GY. Acta Metall 1973;21:1353

[11] Narayan J, Zhu YT. Appl Phys Lett 2008;92:151908

[12] Wu XL, Narayan J, Zhu YT. Appl Phys Lett 2008;93:031910

[13] Jiménez J, Frommeyer G. Mater Charact 2010;61:221

[14] Barbier D, Gey N, Allain S, Bozzolo N, Humbert M. Mater Sci Eng A 2009;500:196

[15] Vercammen S, Blanpain B, Cooman BD, Wollants P. Acta Mater 2004; $52: 2005$

[16] Yan K, Carr D, Callaghan M, Liss K, Li H. Scripta Mater 2010;62:246

[17] Eshelby JD. Proceedings of the Royal Society A: Mathematical, Physical and Engineering Sciences 1957;241:376

[18] Hutchinson JW. Proc Royal Soc A: Math, Phys Eng Sci 1970;319:247

[19] Chun YB, Davies CHJ. Mater Sci Eng A 2011;528:3489

[20] Turner P, Tomé C. Acta Metallurgica et Mater 1994;42:4143

[21] Clausen B, Tome CN, Brown DW, Agnew SR. Acta Mater 2008;56:2456

[22] Cai S, Daymond MR, Holt RA, Oliver EC. Acta Mater 2011;59:5305

[23] Prakash A, Hochrainer T, Reisacher E, Riedel H. Steel Research Int 2008; 
[24] Tome CN, Lebensohn RA, Kocks UF. Acta Metall et Mater 1991;39:2667

[25] Kalidindi SR. J Mech Phys Sol 1998;46:267

[26] Kalidindi SR. Int J Plast 2001;17:837

[27] Bleck W, Phiu-On K, Heering C, Hirt G. Steel Research Int 2007;78:536

[28] Gallagher P. Metall Trans 1970;1:2429

[29] Jones NG, Ward-Close CM, Brown PM, Dye D. Scripta Mater 2010;63:85

[30] Hammersley A, Svensson S, Thompson A. Nucl Inst Meth A 1994;346:312

[31] Lutterotti L. Twelfth Int Conf Text Mater 1999;

[32] Kallend JS, Kocks UF, Rollett AD, Wenk HR. Mater Sci Eng A 1990;132:1

[33] Hill R. J Mech Phys Sols 1967;15:79

[34] Hill R. J Mech Phys Sols 1965;13:89

[35] Ledbetter H, Kim S. Acad Press, New York 2001

[36] Idrissi H, Renard K, Ryelandt L, Schryvers D, Jacques PJ. Acta Mater 2010;58:2464

[37] Bouaziz O, Guelton N. Mater Sci Eng A 2001;319:246

[38] Clausen B, Lorentzen T, Bourke MAM, Daymond MR. Mater Sci Eng A $1999 ; 259: 17$

[39] Rahman K M, Vorontsov V, Dye D. Acta Mater 2015;89:247

[40] Xu F, Holt RA, Oliver EC, Daymond MR. J Neut Res 2007;15:121

[41] Brown DW, Agnew SR, Bourke MAM, Holden TM, Vogel SC, Tomé CN. Mater Sci Eng A 2005;399:1

[42] Yang P, Xie Q, Meng L, Ding H, Tang Z. Scripta Mater 2006;55:629

[43] Idrissi H, Renard K, Schryvers D, Jacques PJ. Scripta Mater 2010;63:961 

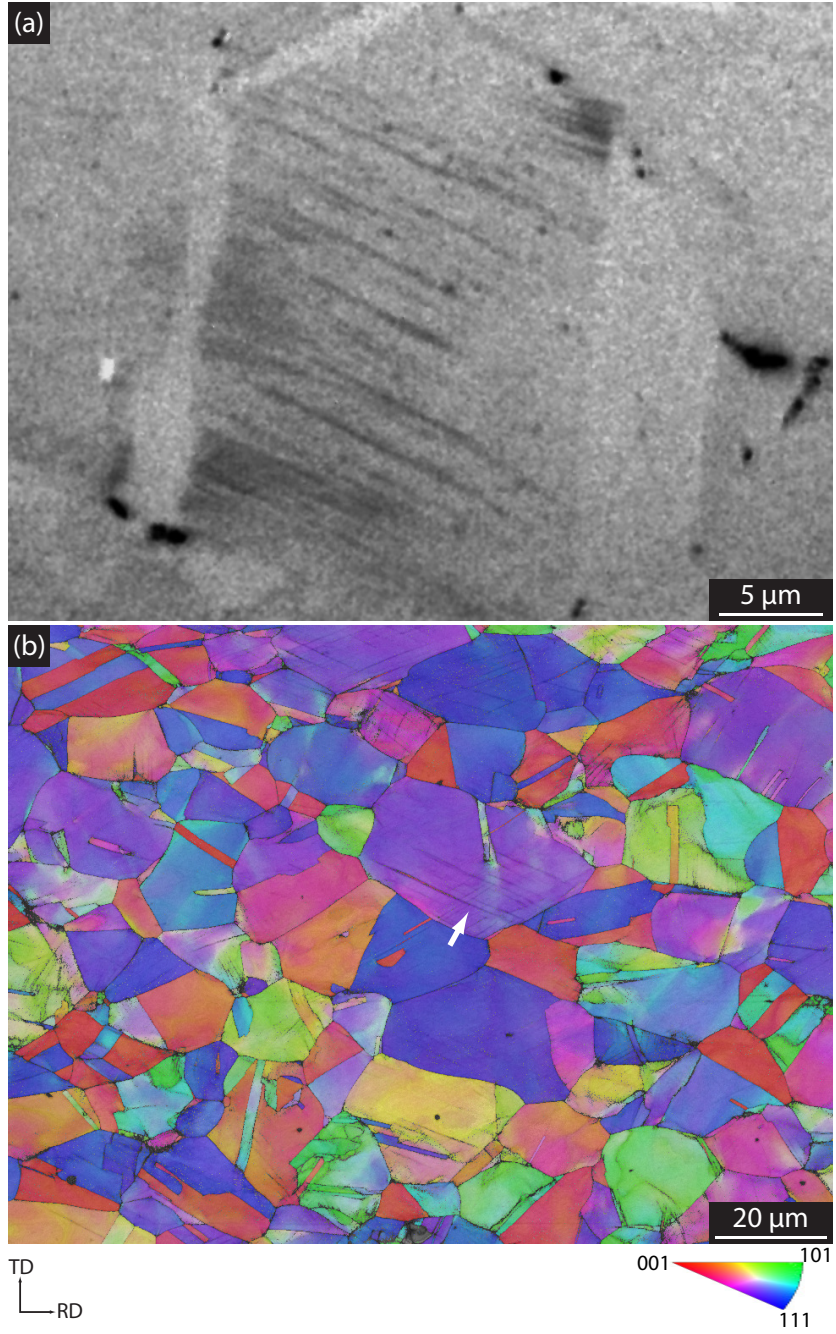

Figure 10: Post deformation microstructure; (a) light micrograph and (b) EBSD map of sample deformed to $22 \%$ strain in the rolling direction with IPF colouring relative to the RD and band contrast. 

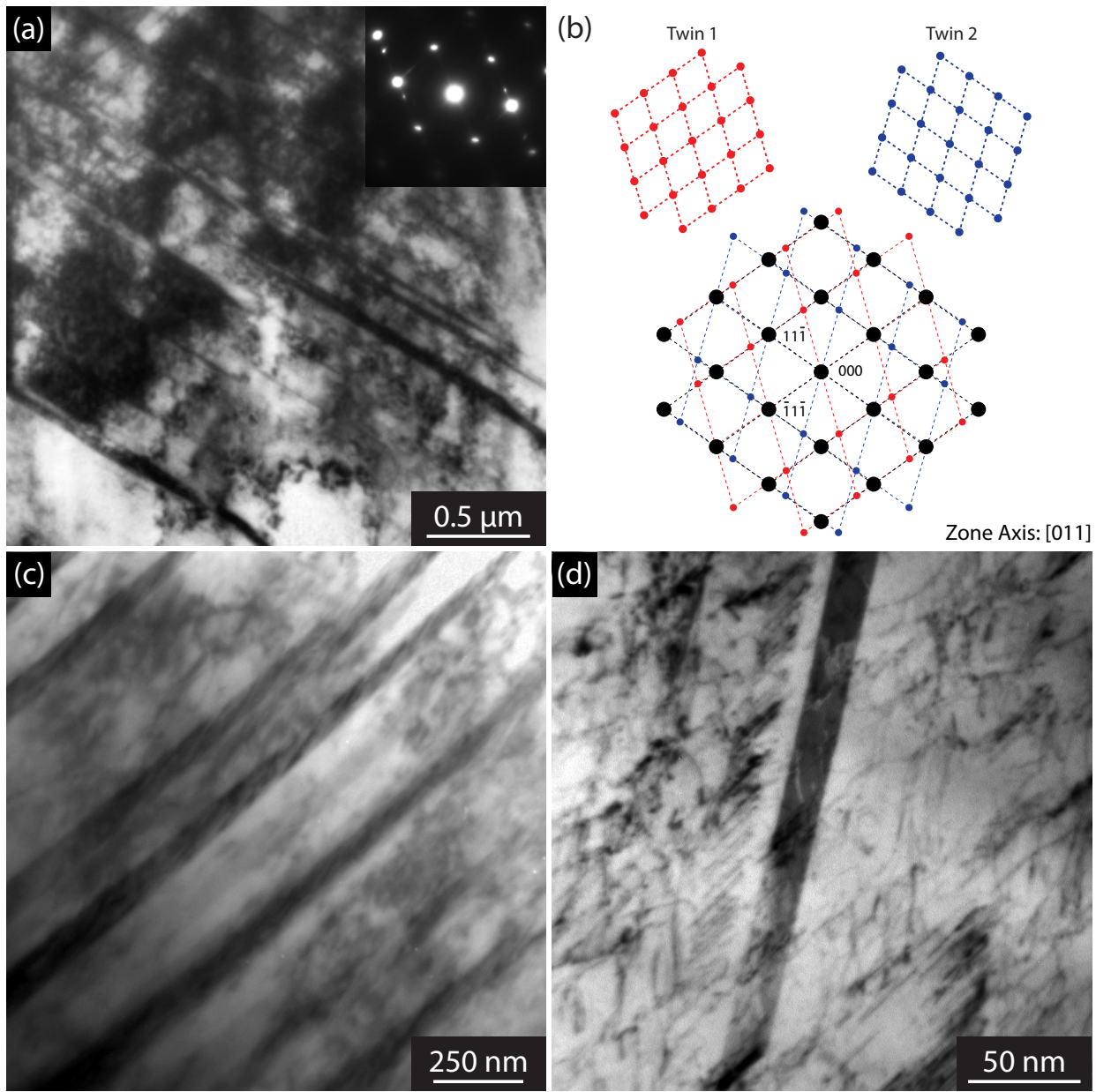

Figure 11: TEM bright field taken from the sample strained to $22 \%$ (a) low magnification with SAED pattern of the grain while the sample is tilted onto the [011] zone axis, (b) schematic of the SAED pattern shown in (a), (c) high magnification of twins and (d) microtwins within a larger twin. 


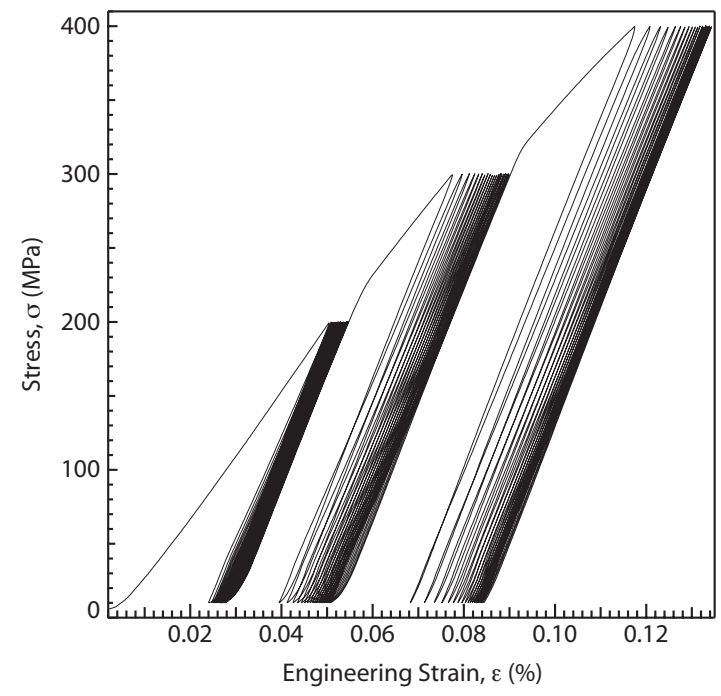

Figure 12: Cyclic tensile loading using an extensometer at below macroscopic yield stresses, indicating the accumulation of strain with each loading cycle.



Figure 13: Single loading and unloading ramp indicating substantial hysteresis occurring and some relaxation. 

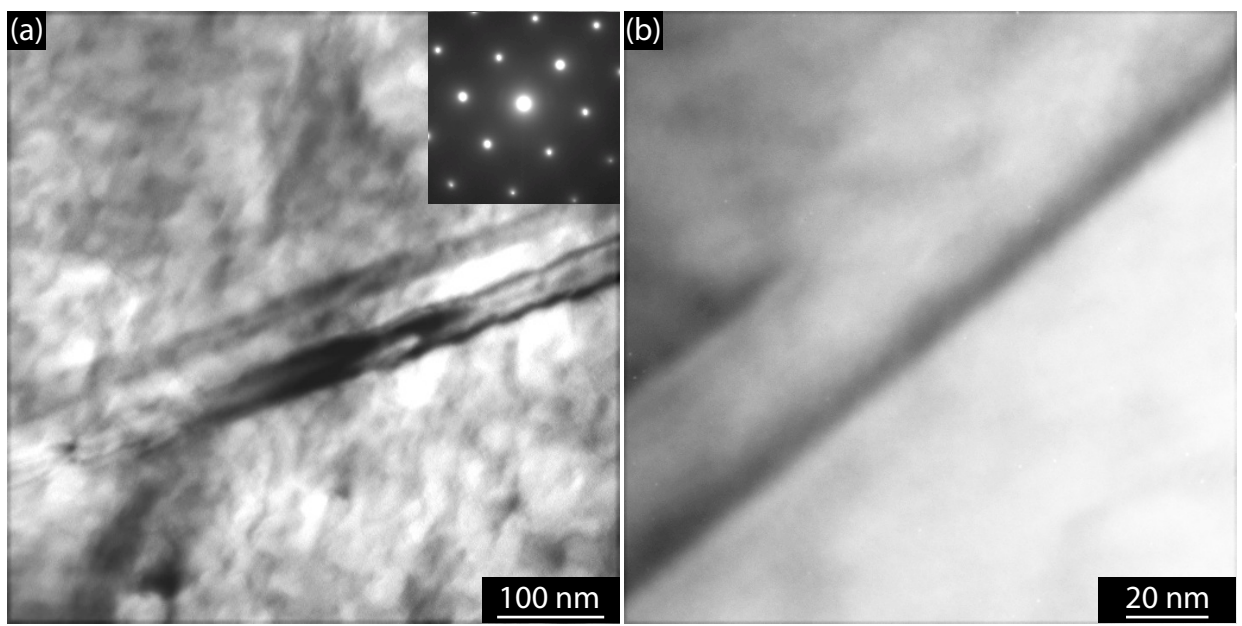

Figure 14: TEM bright field images taken from the sample after cyclic loading (a) fine twin decomposing into stacking faults with SAED pattern taken on the [011] zone axis (g $\{111\})$ and (b) ultra fine twins. 